\title{
Genetic diversity and relationships among different tomato varieties revealed by EST-SSR markers
}

\author{
N.K. Korir ${ }^{1}$, W. Diao ${ }^{2}$, R. Tao ${ }^{1}$, X. Li ${ }^{1,3}$, E. Kayesh ${ }^{1}$, A. Li ${ }^{1}$, W. Zhen ${ }^{1}$ and \\ S. Wang ${ }^{2}$ \\ ${ }^{1}$ College of Horticulture, Nanjing Agricultural University, Weigang, Nanjing, \\ China \\ ${ }^{2}$ Vegetable Research Institute, \\ Jiangsu Provincial Academy of Agricultural Sciences, Nanjing, China \\ ${ }^{3}$ Zhejiang Academy of Agricultural Sciences, Hangzhou, China \\ Corresponding author: S. Wang \\ E-mail: wangsbpep@163.net
}

Genet. Mol. Res. 13 (1): 43-53 (2014)

Received January 3, 2013

Accepted November 7, 2013

Published January 8, 2014

DOI http://dx.doi.org/10.4238/2014.January.8.3

\begin{abstract}
The genetic diversity and relationship of 42 tomato varieties sourced from different geographic regions was examined with EST-SSR markers. The genetic diversity was between 0.18 and 0.77 , with a mean of 0.49 ; the polymorphic information content ranged from 0.17 to 0.74 , with a mean of 0.45 . This indicates a fairly high degree of diversity among these tomato varieties. Based on the cluster analysis using unweighted pair-group method with arithmetic average (UPGMA), all the tomato varieties fell into 5 groups, with no obvious geographical distribution characteristics despite their diverse sources. The principal component analysis (PCA) supported the clustering result; however, relationships among varieties were more complex in the PCA scatterplot than in the UPGMA dendrogram. This information about the genetic relationships between these tomato lines helps distinguish these 42 varieties and will be useful for tomato variety breeding and selection. We confirm that the EST-SSR marker system is useful for studying genetic diversity among tomato varieties. The high degree of polymorphism and the large number of bands obtained per assay shows
\end{abstract}


that SSR is the most informative marker system for tomato genotyping for purposes of rights/protection and for the tomato industry in general. It is recommended that these varieties be subjected to identification using an SSR-based manual cultivar identification diagram strategy or other easy-to-use and referable methods so as to provide a complete set of information concerning genetic relationships and a readily usable means of identifying these varieties.

Key words: Genetic diversity; Tomato varieties; SSR; EST-SSR

\section{INTRODUCTION}

The tomato, Solanum lycopersicum, which originated in Latin America, is the second most important vegetable crop and is cultivated throughout the world (Foolad, 2007). Its production in 2010 reached more than 145.5 million tons, harvested from over 4.3 million hectares, with an average yield of $336,000 \mathrm{Hg} / \mathrm{ha}$ (FAO, 2012). The tomato belongs to the family Solanaceae, which consists of approximately 100 genera and 2500 species, including several plants of agronomic importance such as potato, eggplant, pepper, and tobacco (Olmstead et al., 2008). S. lycopersicum has a relatively compact genome among the Solanaceae species, characterized by its diploidy $(2 \mathrm{n}=2 \mathrm{X}=24)$. It is approximately $950 \mathrm{Mb}$ in size, and is one of the most intensively characterized Solanaceae genomes (Arumuganathan and Earle, 1991). There are more than 7500 tomato landraces and varieties successfully bred and grown for various purposes worldwide, and plant variety registration bodies in different countries keep records of most of these germplasms. These tomato genetic resources are important materials for breeding and biotechnology, and determination of their relationships has valuable potential in the tomato industry. The success of tomato genetic resource collection, preservation, exploitation, and utilization in both present and long-term breeding, and production programs depend largely on the knowledge and understanding of the genetic background, diversity, relationships, and identification of these resources.

Genetic diversity in the cultivated tomato is generally low, due to the occurrence of population bottlenecks during the domestication and generation of modern varieties (Rick, 1976). The cultivated tomato (Lycopersicon esculentum) is known to be highly monomorphic at the molecular level although it is phenotypically very diverse. During and following domestication, the tomato has undergone intensive selection, and cultivated varieties have narrow genetic diversity relative to other crops. This narrow diversity makes it difficult to identify molecular markers that are polymorphic in modern breeding material. However, a number of polymorphic microsatellite markers generated from database sequences have been successfully used for genotyping tomato cultivars and accessions (He et al., 2003; Smulders et al., 1997; Bredemeijer et al., 1998).

Although a variety is traditionally identified by a set of morphological characteristics, these morphological descriptors do not always allow the quantification of genotypic difference because quantitative character can be altered by environmental factors (Cooke et al., 2003). In contrast, molecular markers such as restriction fragment length polymorphism, random amplified polymorphic DNA (RAPD), amplified fragment length polymorphism, and simplesequence repeats (SSR) can provide an effective tool for variety identification since they are 
independent of environmental variation (Korir et al., 2013). Among the different available marker systems, SSR markers have become an important marker system for variety identification because of their genetic co-dominance, high reproducibility, and multiallelic variation (Powell et al., 1996), in addition to relative abundance and good genome coverage.

SSR also known as microsatellite repeats consist of short nucleotide sequences that are repeated many times in tandem. The number of SSR tandem repeats can vary in a sequence, and many such variants (alleles) can exist in a population (Powell et al., 1996). SSR markers tend to be among the most polymorphic genetic marker types and have been introduced into the process of cultivar and variety identification as well as in pedigree reconstruction and genetic mapping (Holton et al., 2002; Yu et al., 2004a; Celucia et al., 2009), to analyze functional diversity (Senior et al., 1998; Leigh et al., 2003; Dreisigacker et al., 2004), and for comparative mapping (Yu et al., 2004b; Varshney et al., 2005a). Although the identification of SSRs in gene sequences of plants started as early as 1993 (Varshney et al., 2005b), full exploitation of this marker during this period was limited by the amount of sequence data available for SSR analysis, and therefore, only a few genomic SSRs were reported. The increase in the amount of sequence data generated from expressed sequence tag (EST) projects in tomato and several other plant species has facilitated the identification of genomic SSRs in large numbers (Wang et al., 2011). By June 2012, there were over 18,208 SSR markers deposited in the Sol Genomics Network (http//:www.solgenomics.net) and available for public use. In addition, many more are found in other databases and laboratories worldwide (Korir et al., 2013). The generation and characterization of EST-derived microsatellites from the tomato and crossspecies amplification in its closely related species and varieties by SSR markers have been done with a total of 7599 SSR markers being generated by in silico data mining of 83,785 sequences (Shirasawa et al., 2010).

SSR markers have been used in tomato variety identification and genetic diversity analysis. For instance, Bredemeijer et al. (2002) differentiated 468 of 521 European tomato varieties using only 20 SSR markers, while He et al. (2003) and Garcia-Martinez et a1. (2006) both confirmed the applicability of SSR markers for analysis of genetic diversity and variability in tomato varieties. The results of Vosman and Arens (1997) indicate that this technique is indeed as efficient in the identification of tomato cultivars. It is widely accepted that SSR techniques are expensive if the sequence information for designing the primers has not yet been developed. However, for the tomato, the primer sets for SSR analysis have already been developed (Smulders et al., 1997; Bredemeijer et al., 1998; He et al., 2003). Besides, SSR has recently produced highly informative genotyping sets in other crops, such as leafy brassicas (Celucia et al., 2009), among others. Furthermore, Jones et al. (1997) and Wang et al. (2011) indicated the reproducibility of SSRs in closely related species and cultivars. The main objective of this study was therefore to evaluate the genetic diversity and relationship of 42 tomato varieties collected from different countries as revealed by SSR markers.

\section{MATERIAL AND METHODS}

\section{Plant materials}

Seeds from a total of 42 tomato genotypes (Table 1) representing geographically distributed origins were collected from research centers, seed companies, and seed shops in 
China and Kenya and planted in a growth room for 3 weeks before extraction of total genomic DNA for use in this study. The initial origins of these varieties are shown in Table 1. The varieties comprised determinate and indeterminate types, small, medium and large fruit types, and fleshy and juicy fruit variants, among other classifications.

\begin{tabular}{|c|c|c|c|c|c|}
\hline Code & Cultivar name & Origin & Code & Cultivar name & Origin \\
\hline 1 & Jingdanfenyu 2 & Beijing & 22 & Tylka & Kenya/Holland \\
\hline 2 & Cai yu 3 & Beijing & 23 & Assila & Kenya/Holland \\
\hline 3 & Ying fen 8 & Beijing & 24 & Cherry sun gold & USA \\
\hline 4 & Xian ke 1 & Beijing & 25 & Zhaoyan 296 & Jiangsu \\
\hline 5 & Jiang shu 14 & Jiangsu & 26 & Bonnie Besst & USA \\
\hline 6 & Xian ke 6 & Beijing & 27 & Luomanna & Holland \\
\hline 7 & Cai yu 1 & Beijing & 28 & German Johnson & USA \\
\hline 8 & Cai yu 2 & Beijing & 29 & Jina & Holland \\
\hline 9 & Sheng xing guo & Jiangsu & 30 & Jiali & Holland \\
\hline 10 & Qiu zhan 16 & Beijing & 31 & Qinghuangfentianshi & Uknown \\
\hline 11 & Jia hong 4 & Beijing & 32 & Hena & Holland \\
\hline 12 & Jiahong 5 & Beijing & 33 & Cherry super sweet 100 & USA \\
\hline 13 & Suhong 2003 & Jiangsu & 34 & Hezuo 908 & Shanghai \\
\hline 14 & Xinyanlvcaiqiu & Heilongjiang & 35 & Gailiangkaluoyi & Holland \\
\hline 15 & Jingdanhuangyu & Beijing & 36 & Zhaoyan 269 & Jiangsu \\
\hline 16 & Eden & Kenya & 37 & Dihuanghuangying tao & Uknown \\
\hline 17 & CAL-J & Kenya & 38 & Beef steak & USA \\
\hline 18 & Jingdanfenyu 1 & Beijing & 39 & Fushi 3 & Holland \\
\hline 19 & Jingdan 1 & Beijing & 40 & Cherry Gardeners Delight & USA \\
\hline 20 & Huangying 1 & Beijing & 41 & Yellow Pear & USA \\
\hline 21 & Jinman & Beijing & 42 & Fenguan & Jiangsu \\
\hline
\end{tabular}

\section{DNA isolation}

Total genomic DNA of each variety was extracted from young leaves using the modified cetyltrimethylammonium bromide method (Murray and Thompson, 1980; Bousquet et al., 1990) and DNA concentrations were quantified using a Hoefer DyNA Quant 200 (Pharmacia Biotech, Piscataway, NJ, USA), while $0.8 \%$ agarose gels were used to ascertain the quality of DNA. The extracted DNA was then diluted to a final concentration of $30 \mathrm{ng} / \mu \mathrm{L}$ with $1 \mathrm{X}$ TE buffer and stored at $-20^{\circ} \mathrm{C}$ until further use.

\section{SSR primers}

Fifty microsatellite markers were chosen on the basis of their repeat patterns (di-, tri-, tetra-, penta-, and hexanucleotide) among the highly polymorphic primers published by $\mathrm{He}$ et al. (2003). These markers were originally screened from the Solanum genomics network (http://www.sgn.cornell.edu). The primers were commercially synthesized by Shanghai Dinguo (Biotechnology Co., Ltd., Shanghai, China) and used for preliminary amplification of the varietal DNA, where 29 primers (Table 2) that amplified the expected polymorphic bands were selected for further use.

\section{Polymerase chain reaction (PCR) amplification and SDS-PAGE}

The 29 pairs of tomato EST-SSR primers were used to conduct PCR amplification in a 
$20-\mu \mathrm{L}$ reaction system containing $2 \mu \mathrm{L} 30 \mathrm{ng} / \mu \mathrm{L}$ genomic DNA, $0.8 \mu \mathrm{L} 10$ pmol of each primer, $0.1 \mu \mathrm{L} 5 \mathrm{U} / \mu \mathrm{L}$ Taq DNA polymerase, $2 \mu \mathrm{L} 10 \mathrm{X}$ buffer, $1.6 \mu \mathrm{L} 25 \mathrm{mM} \mathrm{MgCl}_{2}$, and $1.2 \mu \mathrm{L} 2.5$ $\mathrm{mM}$ dNTPs. Amplification was performed in an Eppendorf Thermal Cycler using the following temperature cycling parameters: initial denaturation for $5 \mathrm{~min}$ at $94^{\circ} \mathrm{C}$ and 35 cycles of denaturation at $94^{\circ} \mathrm{C}$ for $40 \mathrm{~s}$, corresponding annealing temperature for $40 \mathrm{~s}$, extension at $72^{\circ} \mathrm{C}$ for $1 \mathrm{~min}$, followed by a final extension step at $72^{\circ} \mathrm{C}$ for $10 \mathrm{~min}$. PCR products were subjected to electrophoresis on non-denaturing polyacrylamide gels to check the DNA banding patterns.

\section{Data collection and analysis}

To measure the marker polymorphism, the polymorphism information content (PIC) for each SSR was calculated according to the formula PIC $=1-\sum \mathrm{pi}^{2}$, where $p i$ is the frequency of the $\mathrm{i}^{\text {th }}$ allele for each SSR marker locus in the set of 42 tomato varieties investigated (Weir, 1990). The population genetic parameters were estimated using the PowerMarker V3.25 software (Liu and Muse, 2005), including the number of alleles $\left(N_{\mathrm{A}}\right)$, genotype, observed heterozygosity, genetic diversity $(\mathrm{H})$, and PIC. The genetic similarity estimates between 2 cultivars $i$ and $j$ was estimated according to Nei and $\mathrm{Li}$ (1979), which is defined as $\mathrm{Sij}$ $=2 \mathrm{Nij} /(\mathrm{Ni}+\mathrm{Nj})$, where $N i j$ is the number of bands present in cultivars $i$ and $j$, with $N i$ and $N j$ representing the number of bands present in cultivars $i$ and $j$, respectively. For phylogenetic analysis, data only from the polymorphic SSR loci were analyzed by the NTSYS-pc 2.10 statistical software (Rohlf, 2000). All 42 varieties were clustered based on the estimated genetic distance, and the genetic diversity analysis was carried out with the unweighted pair-group method using arithmetic average (UPGMA) clustering and principal component analysis (PCA) method.

\section{RESULTS}

\section{Genetic diversity revealed by SSR markers}

Of the original 50 microsatellite markers used to test the genetic diversity of 42 tomato varieties, $14(28 \%)$ primers failed to amplify the expected PCR fragments, while $7(14 \%)$ amplified monomorphic banding patterns. The remaining 29 (58\%) markers, which generated polymorphic banding patterns, were used in the analysis of genetic diversity where they yielded amenable and reproducible amplicons in the tomato variety samples leading to the detection of a total of 134 alleles and 134 genotypes (Table 2). The $N_{\mathrm{A}}$ per locus varied from 3 to 6, with an average of 4.6, and amplified 134 genotypes, with an average of 4.6. Sequence analysis of PCR fragments verified that differences in the sizes of PCR fragments were predominantly due to SSR. The maximum $N_{\mathrm{A}}$ was 6 as observed in SLR9, SLR11, SLR19, SLR 24, and SLR28, while the minimum $N_{\mathrm{A}}$ was 3 (SLR3, SLR21). The maximum and minimum number of genotypes also followed this trend. The PIC value of the 29 SSR loci varied widely from 0.17 (SLR8, SLR12, SLR17, SLR29) to 0.74 (SLR4, SLR13, SLR20, SLR26), with an average of 0.45 . These results indicated a good genetic diversity among these tomato varieties.

The $\mathrm{H}$ assay revealed a Nei's H that varied from 0.18 (SLR8, SLR12, SLR17, SLR29) to 0.77 (SLR4, SLR13, SLR20, SLR26), with an average of 0.49; the observed heterozygosity ranged from 0.00 (SLR5, SLR10, SLR11, SLR18, SLR21, SLR24, SLR26) to 0.17 (SLR14), with an average of 0.04 . 


\begin{tabular}{|c|c|c|c|c|c|c|c|c|}
\hline $\begin{array}{l}\text { SSR } \\
\text { code }\end{array}$ & Forward primer sequence & Reverse primer sequence & $\begin{array}{l}\mathrm{Tm} \\
\left({ }^{\circ} \mathrm{C}\right)\end{array}$ & $N_{\mathrm{A}}$ & PIC & $\begin{array}{l}\text { Genotype } \\
\text { (No.) }\end{array}$ & $\mathrm{H}$ & $H_{\mathrm{O}}$ \\
\hline SLR1 & f: caa cag cat agt gga gga gg & $\mathrm{r}:$ tac att tct ctc tct ccc atg ag & 56 & 5 & 0.35 & 5 & 0.37 & 0.02 \\
\hline SLR2 & f: tgt tgg ttg gag aaa ctc cc & r: agg cat tta aac caa tag gta gc & 56 & 4 & 0.46 & 4 & 0.54 & 0.02 \\
\hline SLR3 & f: gca cga gca cat ata gaa gag aat ca & r: cca ttt cat cat atc tct cag ctt gc & 56 & 3 & 0.56 & 3 & 0.63 & 0.02 \\
\hline SLR4 & f: act gca ttt cag gta cat act ctc & r: ata aac tcg tag acc ata ccc tc & 56 & 5 & 0.74 & 5 & 0.77 & 0.05 \\
\hline SLR5 & f: ccc aaa tgc tat gca ata cac & r: agt tca gga ttg gtt taa ggg & 56 & 4 & 0.51 & 4 & 0.58 & 0.00 \\
\hline SLR6 & f: tga gaa caa cgt tta gag gag ctg & r: cgg gca gaa tct cga act c & 58 & 4 & 0.46 & 4 & 0.51 & 0.07 \\
\hline SLR7 & $\mathrm{f}$ : tcc aat ttc agt aag gac cce tc & r: ccg aaa acc ttt get aca gag tag a & 58 & 5 & 0.35 & 5 & 0.37 & 0.10 \\
\hline SLR8 & $\mathrm{f}: \operatorname{tgc}$ cca tga cgt tcc atc & r: gac aga cag aga gac aga ctt aga $g$ & 60 & 4 & 0.17 & 4 & 0.18 & 0.07 \\
\hline SLR9 & f: cct ctc ttc acc tct tta caa ttt cc & r: cac tgg tca tta agt cta cag cc & 58 & 6 & 0.50 & 6 & 0.57 & 0.02 \\
\hline SLR10 & $\mathrm{f}:$ aga att ttt tca tga aat tgt cc & $\mathrm{r}:$ tat tge gtt cca ctc cet ct & 58 & 5 & 0.55 & 5 & 0.60 & 0.00 \\
\hline SLR11 & f: gct ctg tcc tta caa atg ata cct cc & $\mathrm{r}$ : caa tgc tgg gac aga aga $\mathrm{ttt}$ aat $\mathrm{g}$ & 58 & 6 & 0.35 & 6 & 0.37 & 0.00 \\
\hline SLR12 & f: gat gga cac cct tca att tat ggt & r: tcc aag tat cag gca cac cag c & 58 & 4 & 0.17 & 4 & 0.18 & 0.02 \\
\hline SLR13 & f: gcc acg tag tca tga tat aca tag & r: gcc tcg gac aat gaa ttg & 60 & 5 & 0.74 & 5 & 0.77 & 0.05 \\
\hline SLR14 & f: gag tca aca gca tag tgg agg agg & r: cgt cgc aat tct cag gca tg & 56 & 5 & 0.35 & 5 & 0.37 & 0.17 \\
\hline SLR15 & f: gga ttg tag agg tgt tgt tgg & $\mathrm{r}: \mathrm{tt}$ gta att gac $\mathrm{ttt}$ gtc gat $\mathrm{g}$ & 60 & 4 & 0.46 & 4 & 0.54 & 0.02 \\
\hline SLR16 & f: cgg cgt att caa act ctt gg & r: gcg gac ctt tgt ttt ggt aa & 58 & 4 & 0.51 & 4 & 0.58 & 0.07 \\
\hline SLR17 & f: ccg cet ctt tca ctt gaa c & r: cca gcg ata cga tta gat acc & 58 & 4 & 0.17 & 4 & 0.18 & 0.02 \\
\hline SLR18 & f: cga tta gag aat gtc cca cag & r: tta cac ata caa ata tac ata gtc tg & 58 & 4 & 0.51 & 4 & 0.58 & 0.00 \\
\hline SLR19 & f: agc cac cca tca caa aga tt & r: gtc gca cta tcg gtc acg ta & 58 & 6 & 0.50 & 6 & 0.57 & 0.02 \\
\hline SLR20 & f: ttc ggt tta ttc tgc caa cc & r: gcc tgt agg att ttc gec ta & 58 & 5 & 0.74 & 5 & 0.77 & 0.05 \\
\hline SLR21 & f: cct tgc agt tga ggt gaa tt & r: tca agc acc tac aat caa tca & 58 & 3 & 0.56 & 3 & 0.63 & 0.00 \\
\hline SLR22 & f: ttg gta att tat gtt cgg ga & $\mathrm{r}: \mathrm{ttg}$ agc caa ttg att aat aag tt & 52 & 4 & 0.46 & 4 & 0.51 & 0.02 \\
\hline SLR23 & f: aca aac tca aga taa gta aga gc & r: gtg aat tgt gtt tta aca tgg & 54 & 5 & 0.55 & 5 & 0.60 & 0.07 \\
\hline SLR24 & f: agg ttg atg aaa gct aaa tct ggc & r: caa cca cca atg ttc att aca aga c & 52 & 6 & 0.35 & 6 & 0.37 & 0.00 \\
\hline SLR25 & f: tgt aga taa ctt cct agc gac aat c & r: acg gac gga tgg aca aat $g$ & 56 & 5 & 0.35 & 5 & 0.37 & 0.02 \\
\hline SLR26 & f: aac ggt gga aac tat tga aag g & r: cac cac caa acc cat cgt c & 60 & 5 & 0.74 & 5 & 0.77 & 0.00 \\
\hline SLR27 & $\mathrm{f}$ : att get cat aca taa ccc cc & r: ggg aca aaa tgg taa tcc at & 60 & 4 & 0.46 & 4 & 0.51 & 0.07 \\
\hline SLR28 & f: taa ata caa aag cag gag tcg & r: gag ttg aca gat cet tca atg & 54 & 6 & 0.35 & 6 & 0.37 & 0.05 \\
\hline SLR29 & f: acg ctt ggc tgc ctc gga & r: aac ttt att att gcc acg tag tca tga & 58 & 4 & 0.17 & 4 & 0.18 & 0.02 \\
\hline Total & & & & 134 & & 134 & & \\
\hline Mean & & & & 4.6 & 0.45 & 4.6 & 0.49 & 0.04 \\
\hline
\end{tabular}

$\mathrm{Tm}=$ melting temperature; $N_{\mathrm{A}}=$ number of alleles; $\mathrm{PIC}=$ pholymorphism information content; $\mathrm{H}=$ genetic diversity; $H_{\mathrm{O}}=$ observed hetereozygosity.

\section{Genetic diversity patterns}

\section{Cluster analysis}

The estimates of similarity between the germplasms tested ranged from 0.18 to 0.77 indicating a fairly big range, thus signifying that the tomato varieties tested showed some diverse relationships. UPGMA cluster analysis of the 42 tomato varieties using the 29 polymorphic markers resulted in the dendrogram shown in Figure 1, which displayed quite a good fit to the genetic similarity matrix. Five groups could be distinguished by truncating the dendrogram at genetic similarity values of 0.71 and 076. Jingdan 1 and Suhong 2003 were clustered in a solitary group (Group I) with a similarity coefficient of 0.71 , while the other 38 varieties fitted into 4 clusters with a similarity coefficient of 0.76 (Figure 2). Group II contained 5 varieties in 4 subgroups, while Groups III, IV, and V contained 14, 3, and 18 lines, respectively. Group V was further clustered into 2 major subgroups containing 7 and 11 varieties, respectively. These cluster analysis results indicated that classification patterns of these tomato varieties may not follow the traditional classification. 
Genetic diversity and relationships in tomato varieties

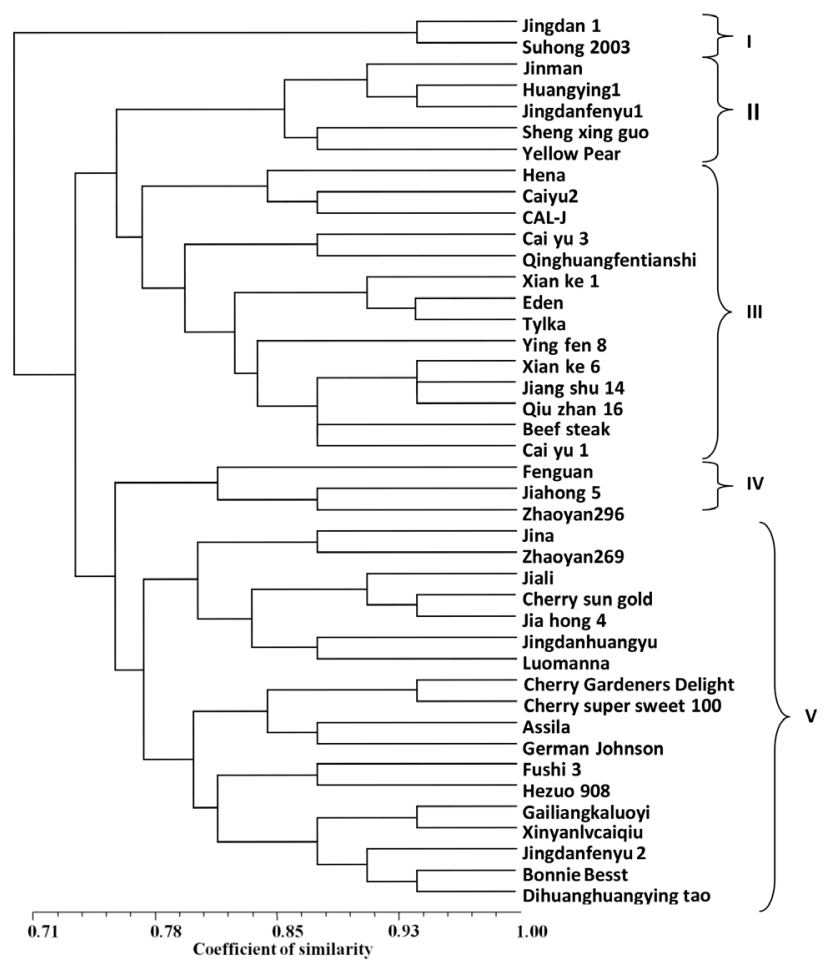

Figure 1. Dendrogram of 42 tomato varieties based on SSR data as clustered using unweighted pair-group method with arithmetic average.

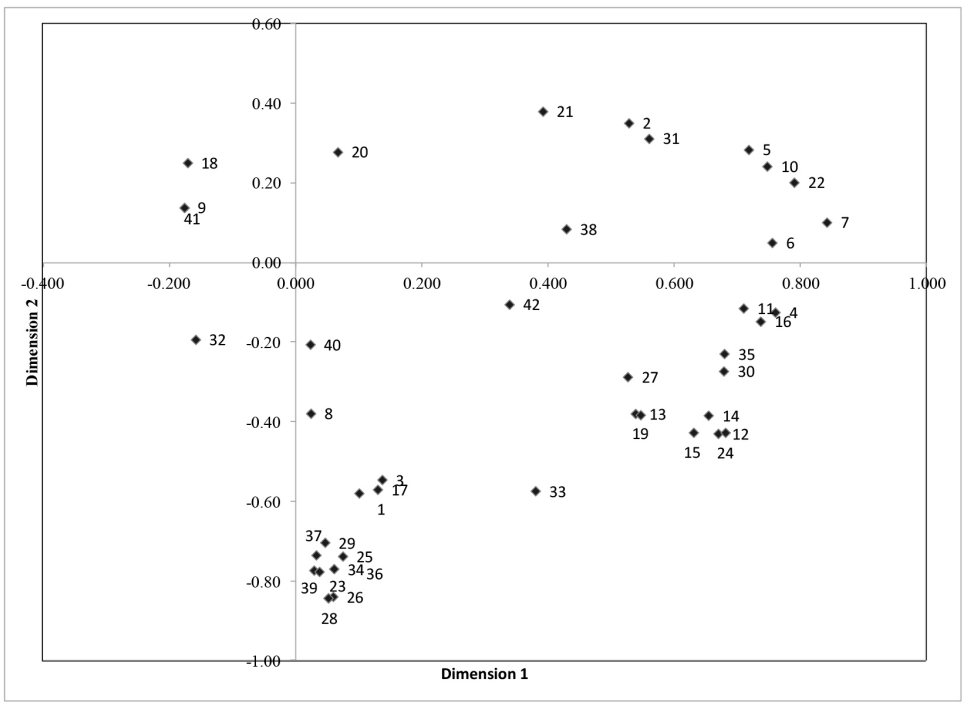

Figure 2. Relationships between tomato varieties as revealed by principal component analysis based on SSR data. The numbers in the figure are code numbers of the 42 tomato varieties detailed in Table 1. 


\section{Principal component analysis}

PCA showed similar results as for clustering, thus confirming the results of UPGMA clustering. A 3-dimensional scatter plot, based on the first, second, and third principal components of the 42 varieties, indicated different levels and directions, so that the degrees of relationships between the varieties could be discerned. Comparison of the UPGMA dendrogram (Figure 2) with the 3-dimensional principal component plot (Figure 2) showed that system clustering and PCA of relationships between genetic resources of tomato and other plants can provide a greater understanding of the complexity of relationship of varieties, cultivars, accessions, and other genotypes in germplasm pools.

\section{DISCUSSION}

DNA polymorphisms within and/or between tomato varieties were investigated on the basis of EST-SSR markers, and variety classification was based on allele frequencies at each locus examined. The $N_{\mathrm{A}}$ per locus at each SSR locus ranged from 3 to 6 , with an average of 4.6, which is comparable to the polymorphisms at SSR loci reported in maize (2 to 13, with an average of 6.5; Labate et al., 2003), tea (2 to 7, with an average of 4.39; Ma et al., 2010), or cucumber ( 2 to 8, with an average of 3.44; Mu et al., 2008), respectively. In addition, the average PIC in this study was 0.45 compared to 0.31 and 0.51 reported in similar studies in other tomato populations (Tam et al., 2005; Benor et al., 2008). The results showed that the tomato varieties tested have a relatively high degree of genetic diversity compared to the generally low diversity among cultivated tomatoes. The $N_{\mathrm{A}}$ recorded in most of the markers used in this study was consistent with another study that employed similar primers (He et al., 2003). The choice of primers used was based on stringent selection with high polymorphism being the key criterion.

The indices used showed that the varieties had a genetic diversity pattern, which is in agreement with the general degree of diversity in cultivated tomato varieties. There were, however, some outstanding materials such as Jingdan 1 and Suhong 2003, which were comparatively distant from other varieties in both the cluster dendrogram and PCA scatter plot. The reasons for this behavior needs further research as it may point to special tomato lines with unique breeding and production value. Compared with previous reports on genetic relationships of tomato germplasm, the 42 varieties studied here had a genetic diversity ranging from 0.18 to 0.77 , which agrees with diversity recorded in other tomato populations (Tam et al., 2005; Benor et al., 2008; Kwon et al., 2009). This could be explained by the fact that most of these varieties were cultivated types from different geographic regions in China and other countries. In studies where the lines compared came from the same area, there is a likelihood of very narrow genetic diversity, just like when cultivated hybrids from the same parental lineage are compared.

Cluster analysis was also effective for variety classification. The classification of the tomato was in accordance with the traditional classification based on morphological traits and, to some extent, the geographical origin, for example, the groups comprising cherry tomatoes, determinate and indeterminate types (Tam et al., 2005) or varieties from Beijing, Jiangsu, Kenya, or Holland, etc. All genetic groups had members with different growth (determinate and indeterminate) and fruiting habits (cherry and non-cherry). As shown in Figure 1, a conspicu- 
ous subgroup similarity was obtained between varieties with the same growth habit (Assila and German Johnson both being indeterminate tomatoes) or fruit type (Cherry Gardeners Delight and Cherry Super Sweet 100 both of which are cherry tomatoes) in Group V. Similar results were obtained for variety classification in tomato using SSR markers (Benor et al., 2008; Kwon et al., 2009; Asgedom et al., 2011; Miskoska-Milevska, 2011), isozymes, and RAPDs (Abd El-Hady et al., 2010). Classification of varieties via cluster analysis based on SSR markers will have a greater advantage because the SSRs have higher polymorphisms and are more discriminative due to co-dominant inheritance compared to either isoenzymes or RAPD markers. Although simple nucleotide polymorphisms and insertion-deletion markers are also informative markers, the phylogenic analysis of SSRs in tomatoes has been shown to be consistent with known pedigrees and previous marker evaluation, while simple nucleotide polymorphisms and insertion-deletions may not reveal clear relationships between populations (Tam et al., 2005).

When PCA and cluster analysis of the SSR results are compared, the results bring out the complexity in the relationship between the varieties. Consequently, studies on the genetic relationships between species should integrate the use of these 2 complementary methods as well as additional strategies to give mutual authentication and subsequently more accurate and reliable results. To make this identification much easier and referable, the construction of an SSR-based manual cultivar identification has been suggested (Zhao et al., 2011).

\section{CONCLUSIONS}

Using 29 selected SSR primer pairs, 42 varieties of tomato from different provinces of China and other countries were evaluated. The average genetic similarity coefficient and PIC indicated a fairly close genetic relationship between these varieties. Similar groups were obtained using UPGMA clustering and PCA methods with the first 3 components in the PCA scatter diagram accounting for a large part of the total variation. Only Jingdan 1 and Suhong 2003 were uniquely classified, while the rest of the accessions were grouped together in 2 main clusters. In addition, the cluster dendrogram indicated no clear differences in geographic distribution characteristics among the 42 varieties studied. It is suggested that a manual cultivar identification strategy be used to generate a cultivar identification diagram from some of the primers, as it may indicate an added potential of EST-SSR markers in plant variety identification. It is recommended that this study be widened to include more varieties, landraces, and other tomato genotypes, as well as a bigger array of markers.

\section{ACKNOWLEDGMENTS}

Research supported by the Fundamental Research Funds for the Central Universities.

\section{REFERENCES}

Abd El-Hady EAA, Haiba AAA, Abd El-Hamid NR and Rizkalla AA (2010). Phylogenetic diversity and relationships of some tomato varieties by electrophoretic protein and RAPD analysis. J. Am. Sci. 6: 434-441.

Arumuganathan K and Earle ED (1991). Nuclear DNA content of some important plant species. Plant Mol. Biol. Rep. 9: 208-218.

Asgedom S, Vosman B, Esselink D and Struik PC (2011). Diversity between and within farmers' varieties of tomato from Eritrea. Afr. J. Biotechnol. 10: 2193-2200.

Benor S, Zhang M, Wang Z and Zhang H (2008). Assessment of genetic variation in tomato (Solanum lycopersicum L.)

Genetics and Molecular Research 13 (1): 43-53 (2014)

CFUNPEC-RP www.funpecrp.com.br 
inbred lines using SSR molecular markers. J. Genet. Genomics 35: 373-379.

Bousquet J, Simon L and Lalonde M (1990). DNA amplification from vegetative and sexual tissues of trees using polymerase chain reaction. Can. J. For. Res. 20: 254-457.

Bredemeijer GMM, Arens P and Wouters D (1998). The use of semi-automated fluorescent microsatellite analysis for tomato cultivar identification. Theor. Appl. Genet. 97: 584-590.

Bredemeijer M, Cooke J, Ganal W, Peeters R, et al. (2002). Construction and testing of a microsatellite database containing more than 500 tomato varieties. Theor. Appl. Genet. 105: 1019-1026.

Celucia SU, De la Peña RC and Villa NO (2009). Genetic characterization of Brassica rapa chinensis L., B. rapa parachinensis (L. H. Bailey) Hanelt, and B. oleracea alboglabra (L. H. Bailey) hanelt using simple sequence repeat markers. Philippine J. Sci. 138: 141-152.

Cooke RJ, Bredemeijer GMM, Ganal MW, Peeters R, et al. (2003). Assessment of the uniformity of wheat and tomato varieties at DNA microsatellite loci. Euphytica 132: 331-341.

Dreisigacker S, Zhang P, Warburton ML, Van Ginkel M, et al. (2004). SSR and pedigree analyses of genetic diversity among CIMMYT wheat lines targeted to different mega-environments. Crop Sci. 44: 381-388.

FAO (2012). Agricultural Statistics of the Food and Agriculture Organization of the United Nations. FAOSTAT | FAO Statistics Division, Rome. Available at [www.fao.org]. Accessed April 9, 2012.

Foolad MR (2007). Genome mapping and molecular breeding of tomato. Int. J. Plant Genomics 2007: 64358.

Garcia-Martinez S, Andreani L, Garcia-Gusano M, Geuna F, et al. (2006). Evaluation of amplified fragment length polymorphism and simple sequence repeats for tomato germplasm fingerprinting: utility for grouping closely related traditional cultivars. Genome 49: 648-656.

$\mathrm{He}$ C, Poysa V and Yu K (2003). Development and characterization of simple sequence repeat (SSR) markers and their use in determining relationships among Lycopersicon esculentum cultivars. Theor. Appl. Genet. 106: 363-373.

Holton TA, Christopher JT, McClure L, Harker N, et al. (2002). Identification and mapping of polymorphic SSR markers from expressed gene sequences of barley and wheat. Mol. Breed. 9: 63-71.

Jones CJ, Edwards KJ, Castiglione S, Winfield MO, et al. (1997). Reproducibility testing of RAPD, AFLP and SSR markers in plants by a network of European laboratories. Mol. Breed. 3: 381-390.

Korir NK, Han J, Shangguan L, Wang C, et al. (2013). Plant variety and cultivar identification: advances and prospects. Crit. Rev. Biotechnol. 33: 111-125.

Kwon YS, Park SG and Yi SI (2009). Assessment of genetic variation among commercial tomato (Solanum liycopersicum L.) varieties using SSR markers and morphological characteristics. Genes Genomics 31: 1-10.

Labate JA, Lamkey KR, Mitchell SE, Kresovich S, et al. (2003). Molecular and historical aspects of Corn Belt dent diversity. Crop Sci. 43: 80-91.

Leigh F, Lea V, Law J, Wolters P, et al. (2003). Assessment of EST- and genomic microsatellite markers for variety discrimination and genetic diversity studies in wheat. Euphytica 133: 359-366.

Liu KJ and Muse SV (2005). PowerMarker: Integrated analysis environment for genetic marker data. Bioinformatics 21: 2121-2129.

Ma JQ, Zhou YH, Ma CL, Yao MZ, et al. (2010). Identification and characterization of 74 novel polymorphic EST-SSR markers in the tea plant, Camellia sinensis (Theaceae). Am. J. Bot. 97: e153-e156.

Miskoska-Milevska E, Popovski ZT, Dimitrievska BR, Bandzo K, et al. (2011). Determination of genetic diversity among different tomato varieties using SSR markers. Acta Agric. Serbica 31: 9-17.

Mu SQ, Gu XF, Zhang SP, Wang XW, et al. (2008). Genetic diversity of Cucumber (Cucumis sativus L.) germplasm by SSR. Acta Hortic. Sin. 35: 1323-1330.

Murray MG and Thompson WF (1980). Rapid isolation of high molecular weight plant DNA. Nucleic Acids Res. 8: 43214325.

Nei M and Li WH (1979). Mathematical model for studying genetic variation in terms of restriction endonucleases. Proc. Natl. Acad. Sci. U. S. A. 76: 5269-5273.

Olmstead RG, Bohs L, Migid HA, Santiago-Valentin E, et al. (2008). A molecular phylogeny of the Solanaceae. Taxon 57: $1159-1181$

Powell W, Machray GC and Provan J (1996). Polymorphism revealed by simple sequence repeats. Trends Plant Sci. 1: 215-222.

Rick CM (1976). Tomato Lycopersicon esculentum (Solanaceae). In: Evolution of Crop Plants (Simmonds NW, ed.). Longman, London, 268-273.

Rohlf FJ (2000). Statistical power comparisons among alternative morphometric methods. Am. J. Phys. Anthropol. 111: 463-478.

Senior ML, Murphy JP, Goodman MM and Stuber CW (1998). Utility of SSRs for determining genetic similarities and relationships in maize using agarose gel system. Crop Sci. 38: 1088-1098.

Shirasawa K, Asamizu E, Fukuoka H, Ohyama A, et al. (2010). An interspecific linkage map of SSR and intronic

Genetics and Molecular Research 13 (1): 43-53 (2014)

CFUNPEC-RP www.funpecrp.com.br 
polymorphism markers in tomato. Theor. Appl. Genet. 121: 731-739.

Smulders MJM, Bredemeijer G, Rus-Kortekaas W, Arens P, et al. (1997). Use of short microsatellite from database sequences to generate polymorphisms among Lycopersicon esculentum cultivars and accessions of other Lycopersicon species. Theor. Appl. Genet. 94: 264-272.

Tam SM, Mhiri C, Vogelaar A, Kerkveld M, et al. (2005). Comparative analyses of genetic diversities within tomato and pepper collections detected by retrotransposon-based SSAP, AFLP and SSR. Theor. Appl. Genet. 110: 819-831.

Varshney RK, Graner A and Sorrells ME (2005a). Genic microsatellite markers in plants: features and applications. Trends Biotechnol. 23: 48-55.

Varshney RK, Sigmund R, Börner A, Korzun V, et al. (2005b). Interspecific transferability and comparative mapping of barley EST-SSR markers in wheat, rye and rice. Plant Sci. 168: 195-202.

Vosman B and Arens P (1997). Molecular characterization of GATA/GACA microsatellite repeats in tomato. Genome 40: 25-33.

Wang YJ, Li XY, Han J, Fang WM, et al. (2011). Analysis of genetic relationships and identification of flowering-mei cultivars using EST-SSR markers developed from apricot and fruiting-mei. Sci. Hort. 132: 12-17.

Weir BS (1990). Genetic Data Analysis-Methods for Discrete Population Genetics Data. Sinauer Associates, Inc., Sunderland.

Yu JK, Dake TM, Singh S, Benscher D, et al. (2004a). Development and mapping of EST-derived simple sequence repeat markers for hexaploid wheat. Genome 47: 805-818.

Yu JK, La Rota M, Kantety RV and Sorrells ME (2004b). EST derived SSR markers for comparative mapping in wheat and rice. Mol. Genet. Genomics 271: 742-751.

Zhao MZ, Zhang YP, Wu WM, Wang C, et al. (2011). A new strategy for complete identification of 69 grapevine cultivars using random amplified polymorphic DNA (RAPD) markers. Afr. J. Plant Sci. 5: 273-280. 